Schweizerisches Jahrbuch für Entwicklungspolitik

26-2 | 2007

Entwicklungsfinanzierung durch Mobilisierung lokaler Ressourcen

\title{
Mikroversicherung als Antwort auf ein Grundbedürfnis
}

Marc Nabeth

\section{CpenEdition}

\section{Journals}

Electronic version

URL: http://journals.openedition.org/sjep/399

DOI: $10.4000 /$ sjep.399

ISSN: 1663-9677

\section{Publisher}

Institut de hautes études internationales et du développement

\section{Printed version}

Date of publication: 1 novembre 2007

Number of pages: 129-148

ISBN: 978-2-88247-069-0

ISSN: $1660-5926$

Electronic reference

Marc Nabeth, « Mikroversicherung als Antwort auf ein Grundbedürfnis », Schweizerisches Jahrbuch für Entwicklungspolitik [Online], 26-2 | 2007, Online erschienen am: 14 Juni 2010, abgerufen am 08 September 2020. URL : http://journals.openedition.org/sjep/399 ; DOI : https://doi.org/10.4000/sjep. 399 


\title{
Mikroversicherung als Antwort auf ein Grundbedürfnis
}

\author{
Marc Nabeth*
}

m gegenseitigen Abhängigkeitsverhältnis zwischen Armut und Verwundbarkeit spielen Einkommensschwankungen eine zentrale Rolle. Sie verstärken bestehende Verwundbarkeiten und perpetuieren so die Armut oder bewirken ein Abrutschen in die Prekarität, sobald unvorhersehbare Ereignisse (Tod eines Familienmitglieds, gesundheitliche Probleme, materielle Schäden usw.) die Einkommenssituation eines Haushaltes belasten. Wie Michel Servet betont, ist Armut aus materieller Sicht weniger das Ergebnis von angesichts der gesellschaftlichen Bedürfnisstruktur knappen Ressourcen als vielmehr die Folge unregelmässiger Einkünfte' .

Indem sie die durch Schadensereignisse hervorgerufenen Einkommensschwankungen abschwächt, kann die Mikroversicherung (d.h. ein Versicherungsangebot, das auf die Situation einkommensschwacher Bevölkerungen zugeschnitten ist) dazu beitragen, Armut und Unsicherheit zu verringern. Das Prinzip ist hinlänglich bekannt: Als Gegenleistung für eine Prämien- oder Beitragszahlung hat der Versicherungsnehmer bei Eintreten des vertraglich vereinbarten Schadensfalles Anspruch auf eine Leistung des Versicherers (Abgeltung in Form von Geld oder Sachleistungen). Diese Verminderung von Ungewissheiten ist für besonders verwundbare Bevölkerungen nicht zu unterschätzen, stellt sie doch zuweilen das letzte Sicherheitsnetz dar, das Bedürftigkeit verhindert, neue Perspektiven eröffnet und eine relative Gewissheit bietet, die Unwägbarkeiten des Lebens meistern zu können.

Dennoch gelten Versicherungen noch immer häufig als Luxus, als ,zusätzliches Standbein", das mit Armut kaum vereinbar ist. Ein Blick auf internationale statistische Kenngrössen wie z.B. die weltweite Verteilung der Beitragszahlungen, die Prämieneinnahmen, die Versicherungsdurchdringung oder die Versicherungsdichte scheint die Ansicht zu untermauern, dass Versicherungen privilegierten Schichten und Gruppen vorbehalten seien (Bevölkerungen der reichen Industrieländer, Wohlhabende in den Entwicklungsländern), (siehe Tabelle).

* Consultant bei CGSI-Consulting (Suresnes, Frankreich) und assoziierter Forscher am Institut Thomas More (Paris und Brüssel).

1 J.-M. Servet, Banquiers aux pieds nus. La microfinance, Paris, Odile Jacob, 2006, S. 51. 
Tabelle : Versicherungen weltweit - ausgewählte Kennzahlen für das Jahr 2005

\begin{tabular}{lll}
\hline 2005 & Industrieländer & $\begin{array}{l}\text { Emerging Markets (Asien, Afrika, } \\
\text { Lateinamerika, Mittlerer Osten } \\
\text { Osteuropa) }\end{array}$ \\
\hline $\begin{array}{l}\text { Weltweite Verteilung } \\
\text { der Beitragszahlungen }\end{array}$ & $12 \%$ \\
\hline Prämien & 1717 Mrd. Dollar (Leben) & \\
\hline $\begin{array}{l}\text { Versicherungsdurchdringung } \\
\text { (direkte Prämien in \% des BIP) }\end{array}$ & 1281 Mrd. Dollar (Nichtleben) $)^{b}$ & 256 Mrd. Dollar (Leben) $)^{b}$ \\
\hline $\begin{array}{l}\text { Versicherungsdichte } \\
\text { (Beiträge pro Einwohner/in) }\end{array}$ & $3,1 \%$ (Leben) $)^{b}$ & $2,2 \%$ (Leben) $^{b}$ \\
\hline
\end{tabular}

Quelle: Swiss Re, Assekuranz Global 2005: moderates Prämienwachstum, ansprechende Rentabilität, Sigma Nr. 5/2005.

a Unter den Schwellenländern wie auch innerhalb der einzelnen Länder sind markante Unterschiede feststellbar.

b Zur Unterscheidung zwischen Lebens- und Nichtlebenversicherung siehe Kasten 1.

\section{Kasten 1: Unterscheidung zwischen Lebens- und Nichtlebenversicherung}

\section{Lebensversicherung}

\section{Todesfallversicherung (Risikoversicherung)}

Beispiel : Die Risikoversicherung garantiert, dass im Falle des Todes des Versicherungsnehmers vor Ablauf der Vertragsdauer eine bestimmte Summe bzw. eine Rente ausbezahlt wird.

\section{Erlebensfallversicherung}

Beispiel: Das garantierte Kapital wird ausbezahlt, wenn der Versicherungsnehmer den Ablauf des Vertrags erlebt. Es handelt sich hierbei um eine aufgewertete Form des Sparens.

\section{Gemischte Versicherung}

Die gemischte Versicherung ist eine Kombination aus Kapitalbildung und Todesfallschutz. Ein Beispiel: Bei einer gemischten Versicherung im Verhältnis 20 : 80 wird dem bzw. den Begünstigtena im Falle des Todes des Versicherungsnehmers vor Ablauf der Versicherung ein Kapital in der Höhe von 20 ausbezahlt. Ist der Versicherungsnehmer bei Ablauf der Versicherung noch am Leben, wird ein Kapital von 80 ausbezahlt.

\section{Nichtlebenversicherung}

\author{
Sachversicherung \\ Beispiele: Diebstahl, Feuer \\ Haftpflichtversicherung \\ Kommt für körperliche oder Sachschäden \\ auf, die der Versicherungsnehmer \\ gegenüber Dritten zu verantworten hat \\ Personenschadenversicherung \\ Beispiele: Pflegeversicherung, \\ Krankenhausversicherung, private \\ Unfallversicherung
}

a Begünstigter: Person, an die die Versicherungsleistung im versicherten Schadensfall ausbezahlt wird.

Die Aufstellung der Swiss Re (siehe oben) unterscheidet zwischen Lebens- und Nichtlebenversicherungen. In Frankreich wie auch in anderen frankophonen Ländern ist diese Unterscheidung nicht sehr gebräuchlich: Dort wird eher zwischen Personenversicherungen und Sach- bzw. Haftpflichtversicherungen unterschieden.

Personenversicherungen decken Schadensereignisse ab, die den Versicherungsnehmer selbst betreffen (Tod, Unfall, Krankheit, Erwerbsunfähigkeit, Invalidität); Sach- und Haftpflichtversicherungen dagegen kommen für die Folgen von Schadensereignissen auf, die die Vermögenswerte des Versicherungsnehmers tangieren (Diebstahl, Feuer, Verlust von Produktionseinrichtungen usw.).

Überlegungen zu möglichen künftigen Entwicklungen sowie eine Rückblende auf die jüngere Vergangenheit legen jedoch nahe, dass dies nicht unbedingt so bleiben muss und die Zahlen nur einen Teil der Realität abbilden. So fragt 
beispielsweise Philippe Trainar, Chefökonom der $\mathrm{SCOR}^{2}$, ob nicht im Kielwasser der Mikrofinanz ein Mikroversicherungswesen im Entstehen begriffen sei, das den Gemeinplatz von der Versicherung als Luxusgut Lügen strafen könnte ${ }^{3}$. Auch Craig Churchill, Vorsitzender der Microinsurance Working Group der Konsultativgruppe zur Unterstützung der Ärmsten (Consultative Group to Assist the Poorest, CGAP), stellt fest, die Versicherer gingen (zu Recht oder zu Unrecht) davon aus, Bevölkerungsgruppen mit geringem Einkommen verfügten nicht über die Mittel, sich zu versichern. Dies überrasche umso mehr, als Ende des 19. Jahrhunderts, als sich Versicherungen zu verbreiten begannen, diese hauptsächlich als Finanzdienstleistung für Arme galten, während Reiche keinen Bedarf danach verspürten, da sie sich aus eigener Kraft absichern konnten ${ }^{4}$.

Das Mikroversicherungsgeschäft weist aber nicht nur Parallelen zur Vergangenheit auf, sondern wirft auch ein neues Licht auf die Gegenwart. Die Mikroversicherung zeige deutlich, dass die für Menschen in den entwickelten Ländern bzw. für Begüterte in den Entwicklungsländern angebotenen Deckungslösungen den Bedürfnissen der einkommensschwachen Bevölkerungen in diesen Ländern in keiner Weise gerecht würden, so Philippe Trainar ${ }^{5}$. In gewissem Sinne gilt diese Feststellung auch für die neuen postindustriellen Gesellschaften mit ihren Beschäftigungskrisen, den sich vergrössernden Ungleichheiten zwischen den verschiedenen soziokulturellen Gruppen und der aleatorischen Einkommensentwicklung, mit denen sich die Einzelnen im Laufe ihres Erwerbslebens konfrontiert sehen ${ }^{6}$.

\section{Kein Allerheilmittel}

Ganz abgesehen von der Verwundbarkeitsproblematik wirft das Mikroversicherungswesen die Frage auf, wie es um die Rechtsstaatlichkeit und die ethischen Grundlagen einer Gesellschaft bestellt ist. Was die Rechtsstaatlichkeit betrifft, so vermag die Mikroversicherung die Verwundbarkeit in kriegsgeschüttelten oder unter einem totalitären Regime leidenden Gebieten nicht zu mildern. Sie ist ebenso wenig wie die Mikrofinanz - ein Allerheilmittel gegen die krasse Armut in Darfur oder in Nordkorea. Aus ethischer Sicht wiederum drängt sich die Frage nach der vom Staat und den Wirtschaftsakteuren getragenen oder geteilten Mutualisierung auf. Natürlich darf dabei die Versicherungsfunktion nicht mit dem Grundsatz der sozialen Umverteilung verwechselt werden. Vielmehr geht es darum, festzustellen, welche Bereiche innerhalb einer Gesellschaft mutualisiert sind. Könnte das Nichtvorhandensein einer gemeinschaftlichen Absicherung für einen Grossteil der Bevölkerung (in den Entwicklungsländern) nicht ebenso ein Zeichen mangelnder demokratischer Reife sein wie ein Absicherungssystem, das

\footnotetext{
Die SCOR ist der grösste französische Rückversicherer.

P. Trainar, „Préface“, in M. Nabeth, Micro-assurance. Défis, mise en place et commercialisation, Les Fondamentaux de l'assurance, Paris, Dalloz; L'Argus de l'Assurance, 2006.

4 C. Churchill, „What Is Insurance for the Poor?“", in C. F. Churchill (ed.), Protecting the Poor: A Microinsurance Compendium, München, Munich Re Foundation; Genf, IAO, 2006, S. 20.

P. Trainar, ibid., S. 13.

6 D. Cohen, Richesse du monde, pauvretés des nations, Paris, Flammarion, 1997. Allerdings ist die Lage in den Entwicklungsländern angesichts der mehr oder weniger stark ausgeprägten Armut, des hohen Bevölkerungsdrucks und der nicht vorhandenen Sozialen Sicherheit für die grosse Mehrheit der Bevölkerung weitaus kritischer.
} 
von der Mehrheit der Bürgerinnen und Bürger in Frage gestellt wird (in den Industrieländern)?

All diese Fragen widerspiegeln sich in gewissem Sinne in den zahlreichen verschiedenen Definitionen der Mikroversicherung, auch wenn letztlich alle ihre Verfechter die Verringerung der Verwundbarkeit der einkommensschwachen Bevölkerungen als gemeinsames Ziel anstreben.

\section{Armut und Mikroversicherung: eine Vielzahl von Definitionen}

\section{Verschiedene Arten von Armut}

Armut ist nicht homogen. Als Resultat eines komplexen sozioökonomischen Prozesses und vor dem Hintergrund des Ausschlusses von Umverteilungsmechanismen oder gesellschaftlicher Differenzierungen (Geschlecht, Alter, Ethnie, Beruf oder Kaste, Dienstalter, Religion, Reichtum usw.) ist Armut ein dynamisches Phänomen, das eine Vielzahl von Verlaufsformen und Situationen umfasst ${ }^{7}$. Anstatt jedoch die fliessenden Übergänge $\mathrm{zu}$ analysieren, werden „Ärmste“, „Arme“, „,mittelmässig Arme“ und „Nichtarme“ abstrakten Objektklassen zugewiesen. Dabei ist das Wissen um die objektiven (von aussen klar erkennbaren) und subjektiven (von den Betroffenen empfundenen) Eigenschaften von Armut für jeden Mikroversicherer eine grundlegende Voraussetzung, denn nur so kann die adäquateste Strategie definiert werden, die dem soziokulturellen Kontext, den Beziehungen zwischen Individuum und Kollektiv und letztlich dem bisherigen Umgang mit Risiken Rechnung trägt.

In Mali beispielsweise wird in der Sprache der Bamanan „Armut“ über den Begriff der Machtlosigkeit (fangantan) wiedergegeben. Im Gegensatz dazu ermöglicht Macht (fangama) zwar den Zugang zu Reichtum, sie verpflichtet aber gleichzeitig den Mächtigen, seine Ressourcen weiterzugeben, da sie als öffentliches Gut gelten. Nur so ist das persönliche Prestige gesichert, das mit dem (zwangsläufig vorübergehenden) Machtbesitz einhergeht ${ }^{8}$. Entsprechend dieser Begrifflichkeit unterscheidet sich die Mutualisierung von Risiken und die Inklusion der Ärmeren im System der Bamanan grundlegend von den Praktiken der Bamiléké in Kamerun: Dort gilt nämlich der Arme als „Glückloser“ (balock), der zudem im Ruf steht, kein ernsthafter Mensch zu sein, und folglich zu meiden ist: Alle Armen stehen im Verdacht, Verschwender zu sein, und laufen Gefahr, aus der Solidaritätsgemeinschaft ausgeschlossen zu werden ${ }^{9}$.

Diese Vielfalt von Konstellationen dürfte der Grund für die Schwierigkeiten sein, sich auf eine Definition von ,einkommensschwachen Bevölkerungen“ und mithin der Mikroversicherung zu einigen. Der Entwurf, den die International Association of Insurance Supervisors (IAIS) im Zuge einer Tagung zum Thema Mikroversicherung im Jahr 2006 erarbeitet hat, illustriert dies: Die IAIS defi-

7 E. Baumann, „Microentreprise et gestion de la vulnérabilité en Afrique subsaharienne, passé et présent“, ADA Dialogue, no 33, 2004, S. 31-53; D. Gentil, „Acteurs, normes et formes de régulation dans la microfinance“, in M. Lévy (dir.), Comment réduire pauvreté et inégalités. Pour une méthodologie des politiques publiques, Paris, IRD; Karthala, 2002, S. 97-124.

8 H. Magassa, „Ethique et pauvreté : l'exemple du Mali“, in collectif, La pauvreté, une fatalité ?, coll. Tropiques, Paris, Karthala, 2002, S. 129-176.

9 J.-P. Warnier, L'esprit d'entreprise au Cameroun, Paris, Karthala, 1993. 
niert die Mikroversicherung als Konstrukt ohne spezifische Produkte oder Produktlinien, das nicht auf einen bestimmten Kreis von Anbietern beschränkt ist, sondern das generell ein Angebot für einkommensschwache Bevölkerungen bezeichnet. Zugleich präzisiert die IAIS mehrfach, dass das Konzept der „einkommensschwachen Bevölkerungen“ je nach Land und abhängig von dessen Entwicklungsstand variieren und die Mikroversicherung je nach Aufsichts- oder Kontrollbehörde unterschiedlich definiert sein kann. In zahlreichen Ländern gilt die Mikroversicherung nicht als eigenständige Versicherungsgattung, sondern schlicht als herkömmliche Versicherung im kleinen Massstab. Dies ist einer der Gründe, weshalb in zahlreichen Gerichtsbarkeiten gesonderte Regelungen zur Mikroversicherung fehlen ${ }^{10}$.

\section{Beispiele für verschiedene Definitionen der Mikroversicherung}

Die Vielzahl der Situationen und Definitionen bleibt nicht ohne Folgen für die Aufgaben und Ziele der Mikroversicherung. Ist sie der Sozialen Sicherheit zuzurechnen oder eher den handelsüblichen Versicherungsdienstleistungen? Richtet sie sich an die Bedürftigen oder eher an den Mittelstand, der über keine soziale Absicherung und über keinen Versicherungsschutz verfügt? Soll sie ausschliesslich den informellen Sektor abdecken oder auch die angestellten Working Poor? Soll sie staatlich kontrolliert oder dem freien Markt überlassen werden? Ist sie als neue Form der staatlichen Subsidiaritätspolitik zu betrachten, bei der die öffentliche Hand die Versicherungsprogramme der lokal und international tätigen wirtschaftlichen und gesellschaftlichen Akteure teilweise subventioniert? Das Feld der Mikroversicherung ist ausserordentlich weit, und entsprechend vieldeutig wird der Begriff in Artikeln, Mitteilungen und Rechtstexten verwendet.

Im südlichen Afrika wird für Versicherungen für Bevölkerungsgruppen mit geringem Einkommen häufig das Prädikat low cost verwendet ${ }^{11}$. In Südafrika richten sich diese Low-Cost-Versicherungen in erster Linie an die Haushalte der Kategorien 1 (niedrigste) bis 5 der LSM (living standards measures). In diesen Haushalten leben rund 17,5 Millionen Erwachsene, von denen 9,5 Millionen monatlich weniger als 500 Rand verdienen.

In Indien ordnet die Indian Regulatory and Development Authority (IRDA) die Mikroversicherung den Lebens- und Nichtlebenversicherungen für einkommensschwache Bevölkerungskategorien im sozialen und ländlichen Sektor zu. Entsprechend der erklärten Politik zur Bekämpfung von Diskriminierung schreibt die Behörde den Privatversicherern hohe Quoten vor ${ }^{12}$ (siehe Kasten 2).

10 International Association of Insurance Supervisors and CGAP Working Group on Microinsurance, Issues in Regulation and Supervision of Microinsurance, November 2006.

11 Vgl. insbesondere die Publikationen des FinMark Trust und des Atlas Magazine. Der FinMark Trust ist eine unabhängige Treuhandgesellschaft, dessen Verwaltungsratsmitglieder aus den Ländern des südlichen Afrikas stammen (<http://www.finmarktrust.org.za>). Das Atlas Magazine wird von Atlas Conseil International herausgegeben, einer in Tunis ansässigen unabhängigen Organisation, die sich auf das Versicherungs- und Rückversicherungsgeschäft in den Schwellenländern spezialisiert hat (<http://www.atlasconseil.com.tu>).

12 M. Nabeth, „Réduire la vulnérabilité des populations 〈pauvres〉 par la microassurance: une synergie entre les Etats et les industriels de l'assurance“, Risques, Nr. 65, März 2006, S. 110-114. 
In Brasilien ist die Volksversicherung - abgesehen von subtilen semantischen Unterschieden - weitgehend mit der Mikroversicherung vergleichbar. Dies zeigen die weit verbreiteten Versicherungsprodukte (Todesfallversicherung der Versicherungsgesellschaft SINAF ab 3,5 Euro pro Monat bei einem garantierten Kapital von 1000 Euro, einer Beteiligung an den Bestattungskosten und Nahrungsmittelhilfe ${ }^{13}$ ), die entstandenen Vertriebspartnerschaften (z.B. zwischen dem Haushaltwarendetaillist Casas Bahia und dem Banken- und Versicherungskonzern Bradesco), die anvisierten Kategorien der unteren Mittelklasse (C und D $)^{14}$ sowie die öffentlichen Interventionen der brasilianischen Aufsichts- und Kontrollbehörde für Privatversicherungen (siehe Kasten 2) ${ }^{15}$.

\section{Kasten 2: Regulierungsbehörden als Wegbereiter}

Die Indian Regulatory and Development Authority (IRDA) und die brasilianische Aufsichts- und Kontrollbehörde für Privatversicherungen (SUSEP) engagieren sich in besonderem Masse für die Förderung der Mikroversicherung. Während Erstere auf reglementarische Vorschriften setzt, bevorzugt Letztere den Weg der steuerlichen Begünstigung.

Indien

Das erste rechtsverbindliche Dokument mit dem Titel Obligations of Insurers to Rural Social Sectors aus dem Jahr 2002 verpflichtet die Privatversicherer, gemäss laufend angepassten Quoten einen gewissen Prozentsatz von Versicherungsverträgen mit "einkommensschwachen“ Kunden abzuschliessen. Ab dem fünften Jahr müssen die Lebensversicherer mindestens $16 \%$ ihrer Policen mit Personen im ländlichen Sektor abschliessen (Ortschaften mit weniger als 5000 Einwohner, Bevölkerungsdichte unter 400 Einwohner pro Quadratkilometer, mindestens $25 \%$ der Erwerbstätigen in der Landwirtschaft) und mindestens 20000 Personen aus dem sozialen Sektor versichern (einschliesslich des informellen und des nicht organisierten Sektors sowie der sogenannt verwundbaren ländlichen oder urbanen Schichten). Die Nichtlebensversicherer ihrerseits müssen mindestens $50 \%$ der Bruttoprämieneinnahmen im ländlichen Sektor erwirtschaften und 20000 Personen aus dem sozialen Sektor versichern.

In einem neueren Text mit dem Titel IRDA (Microinsurance) Regulations 2005 sind verschiedene Grundsätze für das Mikroversicherungsgeschäft festgelegt, darunter solche in Bezug auf die Mikroversicherungsagenten (Kommissionszahlungen, Code of conduct usw.) und auf die Produkte (Arten des Versicherungsschutzes, Höchst- und Mindestdeckung, Dauer des Versicherungsschutzes, Altersgrenzen für den Abschluss von Policen usw.).

Allerdings sind Zweckmässigkeit, Klarheit und Wirksamkeit dieser Regelungen stark umstritten.

Brasilien

Aus der Sicht der SUSEP ist die Mikroversicherung insofern ein wichtiger Geschäftszweig, als sie breite Bevölkerungsschichten einschliesst.

Mit ihrem Rundschreiben Circular SUSEP $n^{\circ} 267 / 2004$ hat die SUSEP für einkommensschwache Bevölkerungsgruppen den Zugang zu Lebensversicherungen erleichtert, indem sie namentlich gewisse Hürden in Bezug auf die Vertriebsnetze (Retail- und Volksbanken, Kirchen usw.) aus dem Weg geräumt hat.

13 Resultat: Innerhalb von weniger als zwei Jahren wurden 300000 Policen abgeschlossen.

14 In Brasilien werden die Haushalte abhängig von ihrem monatlichen Haushaltseinkommen in sieben Einkommensklassen eingeteilt. Haushalte der Klassen A1 und A2 (5,1\% der Bevölkerung) haben ein Monatseinkommen von mehr als 6630 Dollar. Zur niedrigsten Kategorie (E, 2,7\% der Bevölkerung) zählen die Haushalte mit einem monatlichen Einkommen von weniger als 300 Dollar. Zu den Kategorien C und D (zweit- bzw. drittunterste) zählen Haushalte mit einem Monatseinkommen von mehr als 570 Dollar bzw. von mehr als 1140 Dollar (28,5 bzw. 39,3\% der Bevölkerung).

15 Moacir Lamha Filho, Geschäftsführer der SUSEP, „Inclusion sociale de 1'assurance“, $3^{\text {e }}$ Forum international du Centre des hautes études d'assurances, Paris, CHEA, 5. Dezember 2006; E. Lorenzon, „Le Brésil : futur grand marché de l’assurance ?“, Referat, Paris, CHEA, 5. Dezember 2006. 
Damit ist es Priestern nunmehr erlaubt, nicht nur Bibeln zu verteilen, sondern auch Versicherungen anzubieten.

Ein weiteres Rundschreiben (Circular SUSEP $n^{\circ}$ 306/2005) zielt auf die Förderung von Versicherungen für Gebrauchtwagen (10- bis 20-jährige Fahrzeuge) ab, die hauptsächlich von den breiten Volksschichten Brasiliens gefahren werden.

Darüber hinaus beabsichtigt die SUSEP, Versicherungen für ländliche Gegenden zu fördern.

In Frankreich wiederum ergriff die Stiftung Entrepreneurs de la Cité als Erste die Initiative für eine solidarische, kollektive und universale Mikroversicherung. Diese Stiftung wurde von verschiedenen Versicherungsunternehmen (AG2R, April Group, CNP Assurances und La Mondiale), Finanzinstituten (La Banque Postale, La Caisse des Dépôts, EVOLEM) sowie Netzwerken zur Förderung von Unternehmensgründungen (Association pour le droit à l'initiative économique ADIE, France active, France initiative, Réseau des Boutiques de gestion) gegründet und bietet Mikroversicherungslösungen für Jungunternehmer bzw. Jungselbstständige an. Die Jungunternehmer (ehemalige Arbeitslose oder Sozialhilfebezüger/-innen) bezahlen dabei nur die Risikoprämie, die Verwaltungs- und Betriebskosten dagegen werden von der Stiftung getragen ${ }^{16}$. Mittlerweile zeichnen sich am Horizont weitere Formen von Mikroversicherungen ab, die kostengünstige, auf die spezifischen Bedürfnisse von Klein- und Kleinstunternehmen (57 bzw. 39\% der Betriebe in Frankreich) sowie von Selbstständigen in besonders prekärer Lage zugeschnittene Garantiemodelle anbieten.

\section{Unterschiedliche Anbieter für unterschiedliche Zielsetzungen?}

Keine Frage: Das Mikroversicherungswesen ist äusserst heterogen. Seine Grundsätze spiegeln sich nicht nur in von Geldgebern und NRO unterstützten mutualistischen Gesundheitsnetzwerken wider, sondern auch in spezifischen Programmen der grossen privatwirtschaftlichen Versicherungskonzerne. In einigen Fällen richtet sich die Mikroversicherung an die Ärmsten unter den Ärmsten (die mit einem kaufkraftbereinigten Einkommen von einem Dollar pro Tag überleben müssen), in anderen wiederum an die Haushalte einer im Entstehen begriffenen oder aufstrebenden Mittelschicht, die über keinerlei Absicherung durch Sozial- oder Privatversicherungen verfügt. Werden Mikroversicherungen zuweilen von Mikrofinanzinstitutionen (MFI) als Ergänzungsprodukte zu Kredit- oder Spardienstleistungen angeboten, so reichen sie in anderen Konstellationen weit über den Rahmen der Mikrofinanz hinaus und sind dann als neue Formen einer Volksversicherung zu betrachten. In zahlreichen Ländern wie Brasilien, Indien und China, in denen die Soziale Sicherheit äusserst lückenhaft ist und nur etwa 5 bis $15 \%$ der Bevölkerung einen privaten Versicherungsschutz geniessen, überrascht dies nicht. Vor dem Hintergrund des gewollten oder unbeabsichtigten Rückzugs des Staates hat sich die Mikroversicherung zudem in den

16 Angeboten wird eine Altersvorsorge gekoppelt mit einer Berufsrisikodeckung für monatlich 21,92 Euro (ohne zusätzliche Gesundheitsversicherung, die für einen alleinstehenden Erwachsenen 17,75 Euro kosten würde). Die maximale Versicherungsdauer dieser Mikroversicherungslösung, die sich als Sprungbrett zu handelsüblichen Versicherungslösungen versteht, beträgt vier Jahre. Die Prämien entsprechen den technischen Kosten ohne Aufpreis. 
vergangenen 20 Jahren als wichtiger Pfeiler der aufstrebenden Volkswirtschaften etabliert. Einige Beispiele dafür - auf die wir bei der Erörterung der Vertriebsnetze zurückkommen werden - sind die Partnerschaften zwischen Frauenvereinigungen und Privatversicherern in Indien (zwischen der MFI Self Employed Women's Association [SEWA] und AVIVA) oder in Kolumbien (zwischen der MFI Women's World Foundation [WWF] und dem Versicherungsunternehmen La Equidad) zu nennen ${ }^{17}$.

\section{Bedürftigkeit, Segmentierung ${ }^{18}$, Mutualisierung}

Gewisse NRO halten die Einschliessung der Bedürftigen in die Mikroversicherung für unverzichtbar und entwickeln teilweise sogar spezielle Programme, so z.B. die französische Vereinigung CIDR (Centre de développement international et de recherche), die in Mnoungou auf den Komoren eine mutualistische Gesundheitsversicherung namens „Sécurité sociale villageoise“ (SSV) errichtet hat. Kommerzielle Versicherer dagegen vertreten mehrheitlich die Ansicht, dass ihre Mikroversicherung keinen Ersatz für eine soziale Absicherung darstellt. Die Bedürftigkeit als Ergebnis des sozialen Ausschlusses fällt ihrer Auffassung nach primär in den Zuständigkeitsbereich des Staates oder der Gemeinden und nicht in jenen eines Marktteilnehmers. Natürlich geht es nicht darum, Bedürftige zu stigmatisieren und wie gewisse übereifrige Verfechter der Mikrofinanz zu unterscheiden zwischen ,guten Armen“ (solche mit geringem Eigenkapital, aber finanzierbaren Projekten wie Junge, Auszubildende, Witwen, Kleinstunternehmer usw.) und „guten Krediten“ (sprich Investitionskrediten) beziehungsweise „schlechten Armen“ (jene, die kein Risiko eingehen wollen, wie z.B. Betagte, oder sich aus einer gewissen Abhängigkeit nicht lösen können) und ,schlechtem Kredit" (Konsumkredit) ${ }^{19}$. Vielmehr soll betont werden, dass die Mikroversicherung zwar Bedürftigkeit vermeiden helfen kann, aber keinen Ausweg daraus bietet. Diese neuen Versicherungsformen unterstützen und fördern die wirtschaftliche und gesellschaftliche Entwicklung, indem sie gewisse Risiken abdecken und zusätzliche Investitionen auslösen, ohne dass Nichtkleinstunternehmer aus dem Feld der Mikroversicherung ausgeschlossen werden.

Aufgrund des Prinzips der Mutualisierung und des Gesetzes der grossen Zahlen ${ }^{20}$ unterscheidet sich die Mikroversicherung ganz entscheidend von dogmatischen Betrachtungsweisen, die das Mikrofinanzwesen ausschliesslich mit Kleinstunternehmen in Zusammenhang bringen. Die Kenntnis über die verschiedenen Bevölkerungssegmente dient nicht so sehr dem Zweck, gewisse Gruppen auszuschliessen, als dazu, das Portefeuille auszugleichen und abzuwä-

17 Die Vertreter der WWF bieten ihren Kundinnen ein Versicherungsprodukt der Equidad an, das im Falle des Todes oder der dauerhaften Invalidität infolge eines Unfalls eine finanzielle Absicherung bietet. In der Regel sind auch die Bestattungskosten gedeckt.

18 Segmentierung bezeichnet die Aufteilung von Risiken auf mehrere Klassen mit je relativ homogenen Risikoniveaus, für die ein entsprechender Tarif gilt.

19 Besonders aufschlussreich in diesem Zusammenhang ist das Kapitel „L'économie est une négociation linguistique“ in B. Maris, L'antimanuel d'économie, Bd. 1, Rosny-sous-Bois (Frankreich), Bréal, 2003.

20 Gesetz der grossen Zahlen: Unter der Annahme, dass Risiken voneinander unabhängig sind und die Population der Versicherten homogen ist, nähert sich die mittlere Versicherungsleistung pro Versicherter mit steigender Anzahl Versicherter der mathematischen Wahrscheinlichkeit der Versicherungsleistung (reine Prämie) an. 
gen zwischen der Vermeidung „,schlechter Risiken“ bzw. ihrer Integration in die Portefeuilles. Soll ein Dorfältester tatsächlich aufgrund seines fortgeschrittenen Alters von der Versicherung ausgeschlossen werden, oder könnte seine Aufnahme vielleicht dazu beitragen, der Mikroversicherung den Weg zu ebnen? Sollte man nicht viel eher - wie es die American International Group (AIG) in Uganda tut - Ausschlussklauseln für AIDS abschaffen, weil man festgestellt hat, dass der Ausschluss HIV-positiver Personen letztlich der Funktionsweise der Mikroversicherung mehr schadet, als wenn man diese Personen versichert ${ }^{21}$ ?

Marc Cazenave zufolge bewirken solche ethische Überlegungen und technische Schwierigkeiten, dass die Versicherer bei der Risikoselektion und Segmentierung relativ flexibel vorgehen. Gleichzeitig weist er darauf hin, dass die Segmentierung kostspielig (Auswahl von Kriterien, Prämienberechnung, aktuarische Modellierung, regelmässige Aktualisierung) und zuweilen auch riskant ist (ist das Segment nicht gross genug definiert, erhöht sich das Fehlerrisiko markant $)^{22}$.

\section{Doppelte Herausforderung: Nachfrage und Bedürfnisse abdecken und verständliche Produkte anbieten}

Die Festlegung eines für Bevölkerung und Versicherer annehmbaren Mutualisierungsperimeters erfordert zwangsläufig eine Analyse der von der Bevölkerung legitimierten sozialen, wirtschaftlichen und politischen Strukturen. Um diesen gerecht zu werden, müssen sich die Versicherer auf die bestehenden und im Entstehen begriffenen Solidaritätsnetze der Volkswirtschaft stützen, die weit mehr umfassen als den Kreis der mehr oder weniger informellen Klein- und Kleinstunternehmen. Gestützt auf die Netzwerke, die sich der Probleme der einkommensschwachen Bevölkerungen annehmen (MFI, Selbsthilfegruppen, NRO, Detailhandelsketten, semiformelle Bestattungsfonds wie z.B. die Great North Burial Society in Südafrika), und mithilfe einer Kommunikation, die sich eines volksnahen Sprachregisters bedient, kann die Mikroversicherung diese Mischung aus Tradition und Innovation verkörpern, die für die Entstehung eines neuen Sektors unverzichtbar ist ${ }^{23}$.

Dazu muss jedoch der Versicherer die Bedürfnisse und Anliegen der Bevölkerung berücksichtigen und danach trachten, zwei Gebote miteinander in Einklang zu bringen:

$\square$ ein Produkt anzubieten, das den Erwartungen und Bedürfnissen der Bevölkerung entspricht. Diese können abhängig von kulturellen, sozioökonomischen, geografischen, geschlechtsbezogenen und weiteren Faktoren variieren ${ }^{24}$;

${ }^{21}$ M. Nabeth, Micro-assurance. Défis, mise en place et commercialisation, Les Fondamentaux de l'assurance, Paris, Dalloz; L'Argus de 1'Assurance, 2006.

22 J.-M. Cazenave, „Les assureurs santé face aux changements d'accès aux données de santé et gestion du risque: une nouvelle donne pour le modèle économique de l'assurance santé ?“, thèse MBA, France, Ecole nationale d'assurances (ENAss), 2005.

23 J.-M. Servet, op. cit.

24 Vgl. hierzu namentlich die verschiedenen Arbeiten von Michal Matul (z.B. Understanding Demand for Micro-insurance in Georgia [Beitrag zum KfW Microinsurance Symposium in Frankfurt vom 21. und 22. Oktober 2004], Warschau, Microfinance Center, 2004) sowie M. Cohen und J. Sebstad, ,The Demand for Microinsurance“, in C. Churchill (ed.), Protecting the Poor: A Microinsurance Compendium, München, Munich Re Foundation; Genf, IAO, 2006. 
$\square$ mit relativ einfachen Produkten in den Markt einzusteigen, um sich nach und nach mit dem Sektor der Mikroversicherung besser vertraut zu machen. Diese Produkte unterscheiden sich natürlich abhängig vom Know-how und den Zielsetzungen des Versicherers.

\section{Erstes Gebot: Bedürfnisanalyse und Wissensvermittlung als Voraussetzung für die Schaffung einer Vertrauensbasis}

Damit sich das Versicherungs- und Mikroversicherungswesen entwickeln kann, muss eine Vertrauensbasis geschaffen werden, und die Bevölkerung - von den verwundbarsten Gruppen bis hin zur unteren bzw. entstehenden Mittelklasse muss vom Mehrwert der angebotenen Leistung überzeugt sein. Dieses Vertrauen in die Versicherung und in den Versicherer will indessen immer wieder aufs Neue verdient werden ${ }^{25}$.

Damit erscheint in Bezug auf das Versicherungswesen ein Paradigmenwechsel oder zumindest eine Bewusstwerdung über die Problematik der „Versicherbarkeit" - angezeigt. Das Problem ist weniger der Mangel an Kapazitäten bzw. an verfügbarem Einkommen oder die fehlende Versicherungskultur in der Bevölkerung als die Fähigkeit der Versicherer, ihre Instrumente anzupassen. Dies sagte Alfredo Honsberg, Generaldirektor von Seguros Azteca, bei der Bekanntgabe der Lancierung von Mikroversicherungsprogrammen in Mexiko und später in verschiedenen weiteren lateinamerikanischen Ländern ${ }^{26}$.

Das ökonomische Modell, das es zu entwickeln gilt, basiert im Wesentlichen auf dem Verständnis der Gesellschaftskulturen oder der sozialen Systeme. Christophe Jaffrelot warnt jedoch vor kulturbezogenen Erklärungsmustern, denn häufig greife dieser erste Reflex zu kurz. Dagegen liessen sich aus der Geschichte der Gesellschaften gewisse Konstanten ableiten, die unter Umständen von Bedeutung seien ${ }^{27}$.

Nicht zuletzt ist die Mikroversicherung Teil eines Prozesses, der im Volk verankerte und verbreitete Praktiken aufgreift und erweitert. Zu Recht haben Studierende der Ecole polytechnique in ihrer Forschungsarbeit festgestellt, dass für die Versicherer die eigentliche Herausforderung nicht darin besteht, in benachteiligten Kreisen eine Rentabilität zu erwirtschaften, sondern darin, in einem informellen Absicherungsrahmen ein klassisches Versicherungsmodell einzuführen nicht als Ersatz für die herkömmliche Absicherung, sondern vielmehr in Anlehnung daran und mit dem Ziel, die Mutualisierungsperimeter zu erweitern und so die Wirksamkeit des Versicherungssystems zu steigern ${ }^{28}$.

25 Vgl. hierzu namentlich die Arbeiten von Viviana Zelizer über die Skepsis der amerikanischen Volksklassen gegenüber Lebensversicherungen, z.B. Morals and Markets: The Development of Life Insurance in the United States, New York, Columbia University Press, 1979.

26 „Insurers aim at <microinsurance〉 market“, The Herald (Mexico Edition), 15. Dezember 2006. Seguros Azteca folgt in diesem Sinne Banamex, eine mexikanische Versicherungstochter von Citigroup.

27 C. Jaffrelot, „Il n’y a pas de miracle“, interview de François Godement et Christophe Jaffrelot, in P. Gauchont (dir.), Inde, Chine, à l'assaut du monde: faut-il en avoir peur? Rapport Antheios 2006, Paris, Presses universitaires de France (PUF), 2006.

28 Y. Dobel, R. Ducasse, C. Dupont, N. Khemakhem, A. Licht und F. Legrand, „La microassurance, interface entre assurance formelle et réseaux informels", projet scientifique collectif, Ecole polytechnique, 2007. 
Das Eingehen auf die Anliegen der Bevölkerung und deren Beteiligung an der Definition der Produkte, aber auch die Legitimität, die die Promotoren der Mikroversicherung von der Unternehmensleitung bis hin zu den Vertriebsnetzen in den Augen der Öffentlichkeit besitzen, sind letztlich ausschlaggebend. Zur Vertrauensbildung stützt sich der indische Lebensversicherer Tata AIG beispielsweise eher auf den Namen „Tata“ (so heisst auch ein grosser indischer Industriekonzern) als auf „AIG“, da dieser weniger geläufig ist. Die von Tata AIG ausgebildeten Mikroagenten (die in der Regel von NRO empfohlen werden) stammen übrigens selbst aus den Zielgruppen der Bevölkerung.

Von zentraler Bedeutung sind auch die Kommunikationskanäle, denn diese müssen für die Bevölkerung bedeutsam sein. Wandertheater, Kino, Wandmalereien, illustrierte Broschüren - der Beispiele gibt es viele, und ihr Einsatz ist keineswegs auf die Entwicklungsländer beschränkt. Jean-François Estienne, ein Kenner des brasilianischen und japanischen Versicherungswesens, erwähnt die von japanischen Versicherern entworfenen Mangas mit pädagogischem Anspruch $^{29}$. Hier wie dort bedienen sich die Versicherer besonders eindringlicher Botschaften, die auf die Solidarität (,Alle für einen, einer für alle“ im Falle von Yeshasvini), das Vertrauen (z.B. bei der polnischen SKOK, die mit dem Lächeln einer jungen Frau für ihre Sparversicherung wirbt), den Schutz (etwa bei Delta Life in Bangladesh, die dank einer von den Eltern abgeschlossenen Versicherung eine Mitgift erhält) verweisen oder an den Humor appellieren (in Brasilien ist der Slogan ,So wird jede Witwe zu einer guten Partie“, mit dem die SINAF für ein Vorsorgeprodukt warb, in aller Munde).

\section{Zweites Gebot: relativ einfache Produkte, die den Marktkenntnissen des Versicherers entsprechen}

Die Entwicklung der Mikroversicherung erfordert gegenseitiges Vertrauen. Dabei zeigt sich aber, dass die Komplexität der Versicherung ihrer „Demokratisierung“ im Wege steht. Angesichts dessen ziehen es gewisse Versicherer vor, nur ein einziges Versicherungsprodukt zu konzipieren und $\mathrm{zu}$ vertreiben. Kommerzielle Versicherer entscheiden sich häufig für eine kollektive und verpflichtende Kreditversicherung, gegebenenfalls mit einer Zusatzdeckung für Unfall und Invalidität. Solche Produkte lassen sich am einfachsten entwickeln und versprechen die höchste Rentabilität. AIG Unganda beispielsweise bietet über ihre 26 Partner-MFI (24 in Uganda und je eine in Malawi und in Tansania) als einziges Produkt eine obligatorische Kreditversicherung mit Todesfall-, Unfall- und Invaliditätsdeckung an. Die indische Tochter des Lebensversicherers Allianz wiederum garantiert Kreditnehmern ihrer Partner-MFI ASA (Activists for Social Alternatives) gegen eine Jahresprämie von 87 Cent die Auszahlung von 370 Dollar an den Begünstigten, wenn der Versicherungsnehmer tödlich verunfallt oder verstirbt. Anfang 2007 zählte dieses Produkt rund 200000 Versicherte.

Mutualistische Versicherer dagegen bevorzugen aus verschiedenen Gründen (Marktkenntnis sowie das Bestreben, ein Produkt für den wichtigsten Grund der

29 J.-F. Estienne, „Une autre définition de la micro-assurance est-elle possible ? A la lumière de l'expérience brésilienne“, Beitrag zur Konferenz „La micro-assurance: d’un monde à l'autre“, Paris, Club de réflexion Haussmann, Caisse d'allocation vieillesse des agents généraux (CAVAMAC), 13. Februar 2007. 
Verarmung von Haushalten anzubieten) eine ,universelle“ und freiwillige Krankenversicherung, gegebenenfalls auch eine Todesfallversicherung ${ }^{30}$.

Vorsorgeprodukte (Versicherungen gegen Tod, Unfall und Invalidität des Versicherten) gekoppelt mit einer selbst bescheidenen Krankheitsdeckung liegen gegenwärtig im Trend und illustrieren die Suche nach dem „goldenen Mittelweg": Die Vorsorge besticht aufgrund ihrer Einfachheit, die Krankheitsdeckung dagegen versteht sich als Antwort auf ein Grundbedürfnis der Bevölkerung.

Die schrittweise Entwicklung weiterer, komplexerer Produktelinien (Sachschaden, Gesundheit und sogar Klimaschäden) zeugen von einer wachsenden Kenntnis des Mikroversicherungswesens.

Auch die Lebensversicherung (namentlich deren Sparkomponente) erfreut sich seitens der Mikroversicherer zunehmender Beliebtheit. Tata AIG in Indien beispielsweise bietet Versicherungsprodukte mit garantiertem Kapital an und die polnische SKOK eine gekoppelte Lebens- und Sparversicherung für Mitglieder von Kreditunionen.

Die Absicht, kapitalbildende Lebensversicherungen anzubieten (häufig in Verbindung mit einer Todesfallversicherung, bei der den Begünstigten im Falle des vorzeitigen Todes des Versicherungsnehmers eine Kapitalleistung ausbezahlt wird), ist als Antwort auf zwei Tatsachen zu verstehen: die Bedeutung des Sparens als Risikoschutz für einkommensschwache Bevölkerungen einerseits und der Wille gewisser Versicherer andererseits, dieses Sparkapital zu kanalisieren.

\section{Die Versicherung als Instrument der Entwicklungsfinanzierung}

\section{Durch versichern und sparen Risiken vermindern}

Dass das Sparen bei einkommensschwachen Bevölkerungen einen hohen Stellenwert besitzt, ist hinlänglich bekannt. Den Experten Marguerite Robinson und Graham Wright zufolge geniesst das Spargeschäft in Mikrofinanzkreisen oberste Priorität. Michel Servet erinnert seinerseits daran, dass in zahlreichen Ländern die Bereitstellung von Sparmöglichkeiten für die grosse Mehrheit der armen Bevölkerung noch wichtiger ist als die Vergabe von Krediten. So zählten zahlreiche Mikrofinanzinstitutionen, die sowohl im Spar- als auch im Kreditgeschäft tätig sind, mehr Sparer als Kreditnehmer, und das Volumen der Spareinlagen übersteige jenes der gewährten Kredite ${ }^{31}$. Der Wille und die Kapazität der armen Bevölkerung zu sparen übersteigt den Rahmen der Mikrofinanz bei weitem. Erinnert sei in diesem Zusammenhang an die grosse funktionale und geografische Vielfalt der Tontinen (gekoppelt an Lotterien oder Versteigerungen, chit funds in Indien, munno mukabi in Uganda usw.), die dem zielgerichteten Sparen oder dem Zurücklegen für schlechte Zeiten (Vorsichtssparen) dienen ${ }^{32}$. Für eine Beteiligung an einer solchen Sparvereinigung können verschiedene soziale und finanzielle Beweggründe ausschlaggebend sein, die sich keineswegs

30 MicroInsurance Centre, Global Study on Microinsurance, 2006, verfügbar unter <http://www.microinsurancecentre.org >.

31 J.-M. Servet, op. cit., S. 258.

32 S. Rutherford, Comment les pauvres gèrent leur argent, Paris, Groupe d'échange et de recherche technologiques (GRET); Karthala, 2002. 
widersprechen ${ }^{33}$. In den meisten Ländern scheint das Streben nach Absicherung das wichtigste Argument zu sein, sich an Tontinen zu beteiligen. Angesichts dieser Feststellung tritt die Notwendigkeit des Sparens, die in der Literatur stets als grundlegender Faktor für die Existenz der Tontinen genannt wurde, in den Hintergrund: In der Tat seien die Kriterien „Bezahlen der Versicherung“ und „regelmässige Teilnahme an Versammlungen“ wichtige konstituierende Merkmale der Tontinen. So wird in zahlreichen Tontinen von jedem neuen Mitglied als Erstes die Begleichung des Mitgliederbeitrags und der Prämie eingefordert ${ }^{34}$.

An der zweifachen Funktion des Sparens (vor allem Absicherung, in zweiter Linie Kapitalertrag) wird die Rolle der Versicherung in der Entwicklungsfinanzierung noch deutlicher.

\section{Die Versicherung als langfristiger und stabiler Finanzierungskanal}

Im Rahmen eines von der Fédération française des sociétés d'assurances (FFSA) und dem Intstitut Thomas More organisierten Kolloquiums betonte FFSA-Präsident Gérard de La Martinière die Rolle der Versicherung bei der wirtschaftlichen und sozialen Entwicklung eines Landes: Sie fördere das Wachstum, indem sie Risiken im Zusammenhang mit der Wirtschaftstätigkeit als Ganzes mutualisiere, die Unsicherheit der Wirtschaftsakteure vermindere und eine langfristige und stabile Finanzierung bereitstelle ${ }^{35}$. Auch Jean-Philippe Thierry, Präsident und Generaldirektor der AGF, unterstreicht die treibende Kraft der Versicherung in Bezug auf die ökonomische und soziale Entwicklung einer Gesellschaft, die auf folgenden drei Pfeilern beruht:

$\checkmark$ der Mutualisierung von Risiken als stabilisierendem Faktor für die Gesellschaft angesichts der Gefahren, die die Gemeinschaft (Naturkatastrophen usw.) oder das Leben des Einzelnen (Feuersbrunst, Unfall) bedrohen;

$\square$ der Verbreitung guter Praktiken zur Risikoverminderung dank Prävention (die Gewährung eines Prämienrabatts, falls der Versicherte gewisse Massnahmen zur Verringerung des versicherten Risikos trifft, oder die Bereitschaft zur Versicherung eines Risikos, wenn im Gegenzug die Behörden Präventionsmassnahmen ergreifen, sind relativ geläufige Praktiken im Versicherungsgeschäft);

$\checkmark$ der Finanzierung der wirtschaftlichen Entwicklung, wobei die Versicherten langfristig zu Investoren werden (Aktien, Obligationen, Immobilien) ${ }^{36}$.

Patrick M. Liedtke, Generalsekretär und Exekutivdirektor der Association de Genève, führt diese Überlegungen weiter und zeigt auf, dass die Versicherung sich in zweifacher Hinsicht positiv auf die Sparquote einer Volkswirtschaft auswirkt: Erstens erhöhe sie die Sparquote ganz allgemein (namentlich dank Lebensversicherungen), woraus sich ausgedehntere Märkte und höhere Investitionen ergäben,

33 E. Baumann, „Microentreprise et gestion de la vulnérabilité en Afrique subsaharienne ...“, op. cit.; I. Guérin, Femmes et économie solidaire, Paris, La Découverte, 2003.

34 S. Ngassam Bertelet, „A propos d'une composante négligée et oubliée: la microassurance dans l'ouest du Cameroun“, Techniques financières et développement, nº 78, März 2005, S. 116-127 (S. 122).

35 Gérard de La Martinière, „Introduction“, in Institut Thomas More, Assurance, réassurance : une autre contribution au développement. Actes du colloque du 14 juin 2005, Working Papers, Nr. 4/FR, Juli 2006, S. 2

36 J.-P. Thierry, Website der AGF <http://www.agf.fr>, Seite über nachhaltige Entwicklung. 
und zweitens senke sie die Quote des unnützen Vorsichtssparens (Geld, das selten auf den Kapitalmärkten investiert wird) und stimuliere Investitionen und Konsum, indem sie gebundenes (unproduktives oder wenig produktives) Kapital freisetze. Folglich verhelfe die Versicherung einer Volkswirtschaft zu mehr Umlaufsvermögen, da die Menschen sich nicht mehr selbst absichern müssen (beispielsweise für den Fall eines Wohnungsbrandes), denn die Versicherungsmechanismen machten aus ruhendem Kapital verfügbares Kapital ${ }^{37}$.

Zwar erfolgt diese Umwandlung von ruhendem Kapital (,totes Kapital“ in den Worten von Hernando de Soto $)^{38}$ in produktives Kapital keineswegs von allein (aufgrund der Schwäche der Investitionsvehikel in zahlreichen Ländern) und in Bezug auf die ersten Spareinlagen der einkommensschwachen Bevölkerung (hauptsächlich aus dem Vorsichtssparen) häufig verzögert, aber die Idee, dass die Versicherung die wirtschaftliche und soziale Entwicklung eines Landes mit lebensnotwendigem Kapital versorgt, gilt nicht nur für reiche Länder.

Der bangladeschische Versicherer Delta Life erläutert die Ziele seiner MikroLebensversicherungsprodukte wie folgt: „Collect small savings from the people of our country and invest the accumulated savings in profitable nation-building enterprises “39. Diesen Gedanken greift auch Erard Moutassié auf, Generalsekretär der Conférence interafricaine des marchés d'assurance (CIMA), wenn er auf den historischen, wirtschaftlichen und gesellschaftlichen Hintergrund der Entstehung der CIMA verweist: Ab 1986 hätten sich alle betroffenen Staaten in einer äusserst schwierigen wirtschaftlichen Lage befunden, die 1994 in die Abwertung der lokalen Währung (den CFA-Franc) mündete. Die meisten Länder seien bei der Neuausrichtung ihrer Wirtschaft und der Mobilisierung externer Finanzmittel von einschlägigen Institutionen unterstützt worden. Sie hätten erkannt, dass auch inländische Sparguthaben produktiv eingesetzt werden sollten, und seien zur Ansicht gelangt, dass der Versicherungssektor neu ausgerichtet werden sollte, da er offensichtlich nicht in der Lage war, seine angestammte Funktion zu erfüllen. Deshalb hätten sie begonnen, sich neben dem Bankensektor - dem sie bis anhin besonderes Augenmerk geschenkt hätten - auch für die Versicherungen zu interessieren, um Sparguthaben und insbesondere langfristige Einlagen der Lebensversicherungen zu mobilisieren ${ }^{40}$.

Die Entwicklung von Mikrolebensversicherungen mit Prämienrückgewähr wird somit nicht nur durch das Anliegen der Verminderung von Verwundbarkeiten vorangetrieben, sondern auch durch das Ziel, inländische Sparguthaben (jene des informellen und halbformellen Sektors sind nicht zu unterschätzen) zu mobilisieren. Solche gemischte Lebensversicherungen bieten sich als Alternative zu A-fonds-perdu-Produkten an, denen die Bevölkerung in der Regel ablehnend gegenübersteht: Im Erlebensfall erhält der Versicherte nach Ablauf der Versicherung (z.B. zehn Jahre) eine Kapitalauszahlung in Höhe seiner Einlagen

37 P. M. Liedtke, „L'assurance et son rôle prépondérant dans les économies modernes“, in Institut Thomas More, op. cit., S. 5-11 (S. 8).

38 H. de Soto, Le mystère du capital, Paris, Flammarion, 2005. Siehe ebenfalls die nuancierte Kritik von J. Sgard, „La propriété privée et les lois du capitalisme: 1'approche de H. de Soto“, Problèmes économiques, Nr. 2.889, 21. Dezember 2005, S. 33-39.

39 M. J. McCord and C. Churchill (CGAP Working Group on Microinsurance), Good and Bad Practices in Microinsurance: Delta Life, Bangladesh, Case Study, Nr. 7, Februar 2005, S. 16.

40 E. Moutassié, „L'assurance dans les pays en développement: l'exemple africain“, in Institut Thomas More, op. cit., S. 21-25 (S. 21). 
zuzüglich Zinsen (garantiertes Kapital); verstirbt er noch vor Ablauf der Versicherung, erhalten die Begünstigten einen Pauschalbetrag (Risikoversicherung). Delta Life (Bangladesch), Tata AIG (Indien) und ALMAO (Sri Lanka) bieten derartige Verträge an und haben je eigene Erfahrungen in Bezug auf Nachfrage, Machbarkeit und Rentabilität gemacht.

Diese Produkte mit relativ langer Laufzeit sind indessen komplex und teuer. Sie setzen ein grosses versicherungstechnisches Know-how voraus, namentlich in Bezug auf das Aktiv-Passiv-Management und das Aktuariat. Sie sind einem hohen Inflationsrisiko ausgesetzt, das die Wertschöpfung der Spareinlagen der Versicherer stark beeinträchtigen kann. Die Bildung von Vorsorgekapitalien (technische Rückstellungen) hängt ferner von der Performanz und der Stabilität der Finanzmärkte $\mathrm{ab}$, die keineswegs gesichert sind. Und letztlich erfordern diese Produkte eine kontinuierliche Information der Bevölkerungen. Aus all diesen Überlegungen heraus tendieren die Versicherer dazu, die Versicherungsdauer zu verkürzen.

Diese Einwände bedeuten jedoch nicht, dass das Anbieten von Lebensversicherungsprodukten zum Scheitern verurteilt ist, vorausgesetzt allerdings, dass die Versicherer die Kriterien betreffend Zugänglichkeit, Liquidität und Sicherheiten erfüllen, die auch im Spargeschäft von zentraler Bedeutung sind. Aus diesem Grund sind verlustfreie Rückkaufsmöglichkeiten - namentlich im Schadensfall - sehr zu empfehlen.

Ebenso wichtig sind indessen das Verständnis für Belange des Finanzmanagements, des Umgangs mit Risiken seitens der Bevölkerung und der sozialen und wirtschaftlichen Bedeutung des Sparens: Die Modelle der Länder mit einem stark verwurzelten Banken- und Versicherungswesen lassen sich nicht auf andere Gesellschaften übertragen.

Dass sich die Sparer lieber dem informellen Sektor oder den Tontinen zuwenden und ein ,verstecktes“ Sparen vorziehen, das vor dem Zugriff durch Gläubiger geschützt ist, hat verschiedene Gründe: in der Regel tiefere Vermittlungskosten, einfacherer Zugang bzw. erleichterter Rückzug von Spareinlagen, eine Verzinsung, die zuweilen höher ist als jene der formellen MFI (sofern diese überhaupt befugt sind, Spareinlagen entgegenzunehmen), sowie ein grösseres Vertrauen in gewisse informelle Einrichtungen als in den Bankensektor, dessen Ruf unter Konkursen und Veruntreuungen stark gelitten hat.

\section{Die Mikroversicherung als Bindeglied zwischen lokaler und globaler Ebene}

Die bisherigen Überlegungen zeigen, dass die Versicherung kein Luxusgut ist. Vielmehr bietet sie benachteiligten Bevölkerungsgruppen handfeste Lösungen für den Umgang mit Risiken. Die Mutualisierung von Risiken über die Versicherung kann zuweilen simplistische Abgrenzungen (Ärmste, Arme, Nichtarme) überwinden und so der Heterogenität der Bevölkerungen und der durch Einkommensschwankungen hervorgerufenen Armutsdynamik gerecht werden. Die Verbreitung der Versicherung als Instrument zur Risikosenkung und gegebenenfalls als Investitionsvehikel setzt jedoch eine Berücksichtigung der Gesellschaftskultur und den Aufbau einer Vertrauensbasis zwischen Versicherten und Versicherern voraus. 
Die Mikroversicherung als hybrides Konstrukt, in dem sich fremde Einflüsse, Traditionen und Innovationen vermischen und gegenseitig bereichern ${ }^{41}$, kann nur funktionieren, wenn entschieden Abstand genommen wird von ,imperialistischen“ Ansätzen, die das informelle Versicherungswesen als typisches Merkmal archaischer Gesellschaften abtun. Nur ein vielschichtiges Vorgehen, das soziale, kulturelle, wirtschaftliche, politische, rechtliche und historische Erwägungen in Einklang bringt, führt zum Erfolg.

Mit Blick auf die Versicherten bedeutet dies, dass Produkte gut verständlich erklärt und ihre Funktionsweise klar bewiesen werden müssen (rasche Auszahlung von Versicherungsleistungen). Für die Versicherer heisst dies, dass die Kontrollmassnahmen hinsichtlich Betrugs, moralischen Risikos ${ }^{42}$ und adverser Selektion ${ }^{43}$ von der Bevölkerung akzeptiert werden.

Zwischen den Lagern der Versicherer und der Versicherten, deren Sprache und Interessen zuweilen stark divergieren, spielen Fazilitatoren somit eine Schlüsselrolle.

\section{Die Rolle der Entwicklungsagenturen}

Unter „Fazilitatoren“ sind alle Arten von Organisationen zu verstehen, die sich mit den Lebensbedingungen der einkommensschwachen Bevölkerungen auseinandersetzen und die Versicherer mit ihrem Know-how unterstützen können. Häufig wird in diesem Zusammenhang die Zusammenarbeit mit NRO erwähnt, aber gemäss Jean-Michel Debrat von der Agence française de développement (AFD) können auch staatliche Entwicklungsagenturen einen wichtigen Beitrag leisten. Die AFD beispielsweise finanziert Expertisen und die organisationssoziologische Unterstützung in der Hoffnung, dass bis in rund zehn Jahren überlebensfähige Produzentenorganisationen errichtet werden können. Sobald dieses Stadium erreicht ist, kommen die Versicherer zum Zug. Es ist nicht Aufgabe des Versicherers, die Bevölkerung zu erziehen. Die Versicherer müssen zum richtigen Zeitpunkt auftreten, und zwar in dem Moment, in dem eine solide und im Allgemeinen zahlungsfähige potenzielle Kundschaft vorhanden ist. Sobald der Markt reif ist und diese Organisationen Fuss gefasst haben, können sie aus der Obhut der AFD entlassen werden ${ }^{44}$.

${ }^{41}$ I. Guérin, K. Marius-Gnanou, T. Pairault und J.-M. Servet (dir.), La microfinance en Asie. Entre traditions et innovations, Paris, Karthala, 2005.

42 Moralisches Risiko besteht, wenn der Versicherungsschutz die Einzelnen zu einem Verhalten verleitet, das zum Eintreten des Versicherungsfalles führt oder dessen Wahrscheinlichkeit erhöht (vgl. C. Churchill et al., L'assurance et les institutions de microfinance, Genf, IAO, 2004, S. 223-232, $<$ http://www.microfinance.lu/comas/media/glossaire.pdf $>$ ). Die Versicherten sehen keinen Anlass, durch ihr Verhalten das Risikoniveau zu verringern.

Das erwähnte Glossar enthält Definitionen von Begriffen des Versicherungs- und Mikroversicherungswesens.

43 Adverse Selektion liegt vor, wenn die Versicherten über Informationen zum Risikoniveau verfügen, die für die Versicherer nicht zugänglich sind. Diese Asymmetrie verleitet die Versicherten zur Wahl einer Versicherungsdeckung, die ihren Bedürfnissen entspricht, ohne dass diese dem Versicherer bekannt sind. Konkret bedeutet dies, dass eine Versicherungsdeckung mit einer bestimmten Prämie nur von solchen Personen gewählt wird, deren Risikoniveau mindestens gleich hoch oder höher ist als das Risikoniveau, das mit dieser Prämie abgedeckt wird.

44 „Micro-assurance, assurance, réassurance: des outils efficaces pour le développement“, Gespräch mit J.-M. Debrat, M. Nabeth und M. Vaté, Institut Thomas More, Notes, Nr. 11, Januar 2007, S. 8. 
Die Finanzierung des Wissenserwerbs und die soziologische Begleitung (Unterstützung bei der Errichtung gemeinschaftlicher Strukturen, aber auch Wissenstransfer in Bezug auf Gesellschaftskulturen und Bedürfnisse und Anliegen der Bevölkerungen) durch die Entwicklungsagenturen sind unverzichtbar. Insbesondere ersparen sie dadurch den Versicherern einen erheblichen Zeit- und Mittelaufwand, der von einigen Versicherern als Grund für ihr mangelndes Interesse an der Mikroversicherung genannt wird. Seit einigen Jahren entstehen Partnerschaften zwischen Versicherern, Entwicklungsagenturen und Mikrofinanzorganisationen bzw. lokalen NRO mit dem Ziel, Mikroversicherungsprogramme zu entwickeln.

Die Deutsche Gesellschaft für Technische Zusammenarbeit (GTZ) und die Allianz, die bereits in Indien als Partner auftreten, haben in Indonesien gemeinsam ein Pilotprodukt namens „Payung Keluarga“ konzipiert und entwickelt. Für den Vertrieb des Produktes, die Information der Kundinnen und Kunden über das Versicherungswesen, die Prämienfakturierung und sogar für die Auszahlung von Versicherungsleistungen dagegen sind in erster Linie Partner-MFI zuständig, beispielsweise Banken in ländlichen Gebieten, dörfliche Kreditinstitutionen, Spar- und Leihgenossenschaften und NRO. Dank ihrer Stellung und ihres Know-hows über einkommensschwache Bevölkerungen sind diese MFI hervorragend positioniert, um als Agenten der Allianz aufzutreten.

Auch die Zurich Financial Services Group hat eine neue Mikroversicherungsinitiative angekündigt. Dabei handelt es sich um das erste Vorhaben, das mit mehreren verschiedenen Tätigkeitsbereichen des Konzerns abgestimmt und gezielt auf benachteiligte Bevölkerungen ausgerichtet ist. Betont wird vor allem die Zusammenarbeit mit dem Internationalen Arbeitsamt (IAA) und der Direktion für Entwicklung und Zusammenarbeit (DEZA), die ihr technisches Fachwissen und ihre solide Kenntnis über die lokalen Bevölkerungen beisteuern ${ }^{45}$.

Die Nutzung von Synergien zwischen Versicherern, Entwicklungsagenturen, Vereinigungen, NRO und MFI sind zum Teil als Antwort auf die Notwendigkeit zu verstehen, lokale und globale Belange miteinander in Einklang zu bringen. Isabelle Guerin zufolge bietet die lokale Ebene Gelegenheit, sich im Detail mit den Problemen und Bedürfnissen der Bevölkerungen auseinanderzusetzen, während im globalen Kontext Einfluss auf die nationalen Debatten und Gesetzgebungen genommen werden $\mathrm{kann}^{46}$. Zwar ist der vorliegende Beitrag in erster Linie auf die lokale Ebene fokussiert, aber es soll nicht unerwähnt bleiben, dass die Mikroversicherung eine umso grössere Wirkung entfalten kann, wenn sie sich in einen weiteren internationalen Rahmen einfügt, der sich an gewissen ökonomischen und politischen Grundwerten orientiert.

\section{Die Rückversicherung}

Mit Blick auf den langfristigen Bestand der Mikroversicherung stellt sich unweigerlich die Frage nach der Rückversicherung. Denn unabhängig von der Qualität der Schulung von Agenten und der Information der Bevölkerung könnte die Auszahlung von Versicherungsleistungen an alle Versicherten - etwa

45 Zurich Financial Services Group, Zurich Increases Focus on Microinsurance, News Release, 1. Februar 2007.

46 I. Guérin, „Microfinance: des risques et des potentialités“, in S. Allemand (dir.), La microfinance n'est plus une utopie, Paris, Autrement, 2007, S. 161. 
im Falle einer Naturkatastrophe - den Versicherer veranlassen, sein Mikroversicherungsprogramm einzustellen.

Im Anschluss an das Erdbeben im indischen Bundesstaat Gujarat von 2001 beispielsweise musste VimoSEWA Versicherungsleistungen im Umfang von 3400000 Rupien (75000 Dollar) auszahlen. VimoSEWA nahm dieses Ereignis zum Anlass, eine Rückversicherung für sein Portefeuille abzuschliessen, umso mehr, als im Anschluss an das Erdbeben die Nachfrage rapide anstieg und sich die Zahl der Versicherten von 30000 auf 92000 erhöhte. Selbstverständlich bestünde die Möglichkeit, Naturkatastrophen von der Versicherungsdeckung auszuschliessen. Dieses von gewissen Versicherern gewählte Vorgehen kann indessen nach einem Schadensereignis, von dem die gesamte Gemeinschaft betroffen ist, unter den Versicherten Unverständnis und Ablehnung hervorrufen. Dadurch würde das Mikroversicherungsgeschäft als Ganzes diskreditiert. Der Einstieg der Munich Re, der Interpolis Re und anderer Rückversicherer in das Mikroversicherungsgeschäft, aber auch die Entwicklung von Versicherungsprogrammen zur Deckung von Schäden aus Naturkatastrophen (Produkte, die klimatisch bedingte Verluste oder Verdienstausfälle decken und auf einen Klimaindex abgestützt sind), ist für dessen Verfechter höchst erfreulich ${ }^{47}$.

\section{Risikoprävention}

Die Arbeit der internationalen Organisationen und der Regierungen ist ebenfalls von zentraler Bedeutung, namentlich im Hinblick auf die Prävention. Michel Jarraud, Generalsekretär der Weltorganisation für Meteorologie (WMO), betont, dass mit jedem Dollar, der in die Prävention investiert wird, 10 bis 100 Dollar an Wiederinstandsetzungskosten nach extremen Naturkatastrophen eingespart werden können ${ }^{48}$. Auch der Ökonom François-Xavier Albouy sieht hier eine zentrale Herausforderung, denn obwohl die Schadenssummen der direkten Folgen von Naturkatastrophen mit durchschnittlich rund zwei Prozent des weltweiten BIP gesamthaft betrachtet relativ niedrig sind, können die Konsequenzen je nach Entwicklungsstand einer Volkswirtschaft stark variieren ${ }^{49}$. Allein für das Jahr 1998 wurden die wirtschaftlichen Einbussen infolge von Naturkatastrophen für Nicaragua und Honduras auf 50 bzw. 37,7 \% des BIP geschätzt. Die Prävention beschränkt sich indessen nicht auf das Naturkatastrophenrisiko: Das Engagement der sozialen Organisationen in den Bereichen Bildung und Gesundheit ist von derart grosser Bedeutung, dass die Versicherer gut daran tun, mit diesen Organisationen partnerschaftlich zusammenzuarbeiten.

47 Von Interesse sind in diesem Zusammenhang die Arbeiten von Michel Vaté, die auf PlaNet Re, $<$ http://www.institut-thomas-more.org> verfügbar sind, z.B. Réassurer la planète. La mondialisation financière au services des plus pauvres, Institut Thomas More, Notes, Nr. 1/FR, 15. Januar 2004, und „L'objectif de sécurité alimentaire: quel rôle pour l'assurance? in Institut Thomas More, Assurance, réassurance: une autre contribution au développement. Actes du colloque du 14 juin 2005, Working Papers, n 4/FR, Juli 2006, S. 44-47. Siehe auch E. de Tayo, „Planet Re: une alternative à l'aide publique au développement par l'assurance“, Gespräch mit Michel Vaté, Afrique Expansion Mag, Nr. 24, 2006.

48 Y. Veyret, „Risques et prévention“, Les catastrophes naturelles, dossier, Questions internationales, Nr. 19, Mai/Juni 2006, S. 12-22, insbesondere S. 13.

49 F.-X. Albouy, „Economie des catastrophes“, Les catastrophes naturelles, dossier, Questions internationales, Nr. 19, Mai/Juni 2006, S. 66-72 (S. 69). 


\section{Die Rolle der Politik}

Trotz der Bedeutung der Rückversicherung stellt sich letztlich die Frage nach der Verantwortung des Staates und der Politik. Diese Frage soll hier nicht zum Anlass für die Forderung genommen werden, über einen öffentlichen Rückversicherer unbegrenzte Deckungen für bestimmte Ereigniskategorien wie Naturkatastrophen oder Terroranschläge anzubieten oder der Mikroversicherung mit Subventionen unter die Arme zu greifen, wie in der ersten Ausgabe 2007 der Zeitschrift Sigma der Swiss Re am Beispiel der Agrarversicherungen illustriert wird. Die Frage nach der Verantwortung des Staates steht in direkter Abhängigkeit zur Rechtsstaatlichkeit. So hilfreich und notwendig die Mikroversicherung auch sein mag, so ist sie nicht in der Lage, die Probleme der Armut allein zu lösen. Die Verfügbarkeit und die gemeinsame Nutzung öffentlicher Güter (Bildung, Gesundheitswesen, kollektive Infrastrukturen) haben einen massgeblichen Einfluss auf die Wirksamkeit der Mikroversicherungsprogramme. Jean-Michel Servet weist darauf hin, dass Armut als Folge hierarchischer Unterschiede zu verstehen ist, in anderen Worten als Ergebnis der Statusunterschiede und der Diskriminierungen, die zu Marginalisierung und Exklusion führen: Je stärker die Gesellschaft eines Landes fragmentiert sei, desto grösser sei der Anteil der Armen an der Gesamtbevölkerung ${ }^{50}$.

Es geht also nicht um die Verwirklichung eines egalitaristischen Ideals, sondern um demokratische Reife.

In seinem Essay La démocratie des autres entkräftet Amartya Sen anhand verschiedener historischer, kultureller oder zivilisatorischer Beispiele die Idee, wonach die Demokratie ein rein westliches Konstrukt sei, und unterstreicht die universelle Geltung des demokratischen Systems und seiner Funktion, Wertvorstellungen zu schaffen.

Indem sie allgemeine Bedingungen für die Konzipierung, die Tarifgestaltung und den Vertrieb von Versicherungsprodukten schafft, die die in den Bevölkerungen verwurzelten Dimensionen des Individuums, des Kollektivs, der Solidarität und der Identität gleichermassen berücksichtigt, trägt die Mikroversicherung dazu bei, die Rolle der Versicherung innerhalb des ökonomischen und sozialen Gefüges eines Landes zu überdenken, und fördert auf diese Weise letztlich die Qualität einer Demokratie ${ }^{51}$.

50 J.-M. Servet, op. cit., S. 463.

51 M. Nabeth, „La micro-assurance: un rôle structurant dans les nouvelles réalités sociales“, Entrepreneurs de la Cité, La Lettre, Nr. 2, Dezember 2005. 


\section{Auswahlverzeichnis}

ADA Dialogue, $\mathrm{n}^{\circ}$ 33, 2004, Thema „La micro-assurance“.

Churchill, C. F. (ed.), Protecting the Poor: A Microinsurance Compendium, Munich, Munich Re Foundation; Genf, IAO, 2006.

Churchill, C. F., D. Liber, M. J. McCord und J. Roth, L'assurance et les institutions de microfinance, Guide technique pour le développement et la prestation de services de micro-assurance, Genf, IAA, 2004.

Nabeth, M., Micro-assurance. Défis, mise en place et commercialisation, Les Fondamentaux de l'assurance, Paris, Dalloz; L'Argus de l'Assurance, 2006.

Roth, J., M. J. McCord und D. Liber, The Landscape of Microinsurance in the World's 100 Poorest Countries, Microinsurance Centre, April 2007.

Servet, J.-M., Banquiers aux pieds nus. La microfinance, Paris, Odile Jacob, 2006.

\section{Internet Adressen}

Allianz: <http://www.knowledge.allianz.com/en/globalissues/microfinance>.

Appui au développement autonome (ADA) : <http://www.microfinance.lu $>$.

Indische Kontroll- und Entwicklungsbehörde für Versicherungen : <http://www.irdaindia.org > .

Concertation entre les acteurs du développement des mutuelles de santé en Afrique: $<\mathrm{http}: / /$ www.concertation.org $>$.

Entrepreneurs de la cité : <http://www.entrepreneursdelacite.org >

Consultative Group to Assist the Poorest (CGAP), Appui au développement autonome (ADA) und Groupe d'échange et de recherche technologiques (GRET) : <http://www.lamicrofinance.org $>$.

Brasilianische Regulierungs- und Kontrollbehörde für Privatversicherungen: <http://www.susep.gov.br/ menuingles/legiseng.asp\#mi>.

Institut Thomas More, Seite zum Thema Entwicklungshilfe: <http://www.institut-thomas-more.org/welcome/ developpement>.

Microinsurance Centre : <http://www.microinsurancecentre.org > .

Munich Re: <http://www.munichre-foundation.org >.

Internationale Arbeitsorganisation (IAO):

Seite „Global Information on Micro-Insurance“ (GIMI) : <http://www.ilo.org/gimi> ;

Seite ,Social Finance Programme“, „Reducing Vulnerability“: <http://www.ilo.org/public/english/ employment/finance/vulnerab/micro/index.htm>.

Strengthening Micro Health Insurance Units for the Poor in India (gemeinnütziges Konsortium) : <http:// www.microhealthinsurance-india.org $>$. 